\title{
Smart Home Automation System for Elderly, and Handicapped People using XBee
}

\author{
Bilal Ghazal ${ }^{1}$ and Khaled Al-Khatib ${ }^{2}$ \\ ${ }^{1}$ Faculty of Sciences IV, Lebanese University (UL), Zahle, Lebanon \\ ${ }^{2}$ Faculty of Engineering, Lebanese International University (LIU), Khyara, \\ Lebanon \\ ${ }^{1}$ Bilal.ghazal@ul.edu.lb, ${ }^{2}$ Khaled.khatib@liu.edu.lb
}

\begin{abstract}
Smart home automation system is increasingly used due to the wide manufacturer brands and various available technologies. From a social point of view, residents are admitted to smart homes for comfort, luxury, improving quality of live, and for providing security against intrusion and burglars. Secondly, home automation is achieved using a single controller, monitoring and the controlling many interconnected appliances such as lights, power plugs, HVAC system, humidity and temperature sensors, gas, smoke and fire detectors, audio, video and home theater as well as security and emergency systems. Smart homes are cheap, low-power, cost effective, efficient, and realize the automation of a variety of domestic appliances using user-friendly interface as remote control or any other handheld devices. Elderly, handicapped patients, and people with disabilities who have problems with locomotion difficulty can benefit from this smart home to totally operate, with high performance, all appliances and devices from anywhere in the house. When a resident is living alone, the ubiquitous access becomes very important and it is realized by using XBee transceivers that maintain $R F$ wireless communication between the remote control and the master control panel board.
\end{abstract}

Keywords: Smart Home; Automation System; Remote Control; Microcontroller; Wireless Communication; XBee

\section{Introduction}

Elderly people are an important and growing segment in the world population. The statistics show that the percentage of older people is continuously growing due to many reasons, in particular, the declining of birth rates and the reduction of women fertility. In the United States, the proportion of population 65 years and over has increased from $12.4 \%$ in 2000 to $13.3 \%$ in 2011 and it is expected to grow to reach $21 \%$ of the population by 2040 [1]. Moreover, the current social life style, modern medicine, and the easy access to medical care have increased life expectancy. A United Nations report [2] estimated that the life expectancy was 65 years in 1950, and 78 years in 2010 and it will continue to rise to 83 years in 2045 .

On the other hand, it was reported that $35 \%$ of people age $65+$ in 2011 had some type of disability [1]. Some of them require assistance to meet important personal needs. Frail older adults prefer living independently and self managing in their own home which promote the feelings of competency and reduce the vulnerability to depression. In fact, from economic point of view, the cost of living at home with monitoring devices and intelligent appliances is less expensive and more beneficial than attending medical centers and being supervised by nurses. However, the implementation of smart home systems with remote monitor controls and health care capabilities will reduce the expense of personal aid assistance at home. 
The aim of this article is to propose a wireless remote control that permits elderly people with physical challenges, in particular, handicapped and disabled people, to command their desired devices without moving around to the nearest control point. Indeed, the local control is not excluded but alternative additional controls are achieved using a remote control supported by XBee communication transceivers. The remote control includes laser-engraved backlit buttons customized to the special needs of elderly users as well as Braille interface for blind persons.

\section{Smart homes}

Smart homes, intelligent homes, home automation, domestics and others are all synonyms that describe, according to the Smart Home Automation of Netherlands, the "integration of technology and services through home networking for a better quality of living" [3]. The UK Department of Trade and Industry defines the smart house as "a dwelling incorporating a communications network that connects the key electrical appliances and services, and allows them to be remotely controlled, monitored, or accessed". The smart home system is not a new science terminology but it is still away from people's vision. In fact, the majority of home appliances are somehow automated but the integration of these technologies, the inter-corporation of automated various appliances in an affordable design, and the ease of deployment due to distant communication provides peace of mind and convenience. These systems are feasible, low-power consumption, secure, efficient, flexible and scalable, cost-saving, characterized by ubiquitous access, and finally supported by easy-to-use familiar interface.

\subsection{Controlled Appliances}

Smart homes involve different areas of electronics, architecture, computing, and communications. A smart home achieves a complete and total control of unlimited number of appliances. It commands the On/Off order of domestic devices such as fridge, $\mathrm{TV}$, washing, cooking, and cleaning machines, as well as electrical devices as motors, pumps in order to water the houseplants using humidity and soil moisture. It governs the environmental system such as HVAC (Heating, Ventilation, and Air Conditioning), and fans. It also masters the lamps arrangement as switching, dimming lights, and making ambiance for different events. It has the capability to control windows blinds and curtains to maximize natural lighting. Moreover, it enables multiple levels of security by implementing gas, smoke, and fire sensors connected to sirens, and by using intrusion detectors and automatic motion detectors outside homes that differentiate pets from intruders. It employs glass breaking detectors, surveillance and camera systems as well as monitoring home structure systems for detecting vibrations and earthquakes using 3D accelerometer sensors. In addition, the home theater and the entertainment system acquire high degree of conformability and permit the users to give the admittance for visitors using video door phone and fulfills regular visitor entry option. Furthermore, the home theater authorizes the diffusion of the favorite audio and video, the broadcasting of music in any part of the building, and the realization of video communication between different rooms.

Finally, smart homes are of great interest since they are water and power saving systems, since their power consumption could be managed using smart meters, temperature auto control, and battery supervising levels. Moreover, it can be supported by solar tracking and wind green energy systems to reduce the pollution injection. Furthermore, smart homes can be configured by scheduling tasks for automatic control settings in order to accomplish more rational use of energy. At the end, the functionality with biometric sensors allows elderly and disabled people to be virtually monitored by 
dedicated health progress programs. The appropriate biometric devices measure the irregular heart rhythms, respiration rate, blood pressure, and skin temperature; notify medication periods and doctors meetings, and alerts hospital in case of emergency.

\subsection{Smart Home Technologies}

Many various technologies [5-22] have been proposed in the literature and depend on the way the control signal is spread from the end-user remote control to reach the target appliances through master control panel boards.

The first technology is the Power-line Carrier system where the control coded signals propagate through the household existing electrical power wiring. While the power line transmits AC signal of frequency $50 \mathrm{~Hz}$ or $60 \mathrm{~Hz}$, the command signals are used in the range of $24 \mathrm{kHz}$ to $500 \mathrm{kHz}$. The major drawbacks are the propagation problems and the electronic interference where noisy signals can be interpreted in a wrong manner.

The second technology pursues the home appliances using landline telephone system (analog telephone service). The procedure is simple: when a dial-up connection is established and maintained for limited time duration, additional digits are dialed up for various controls [7]. Each combination of numbers is associated to a specific appliance or equipment. This mechanism does not require internet connection. Its drawback resides in the unattainable capability to inspect the status of appliances. However, the digital phone service often called VoIP (voice over IP) carries the digital signals over a broadband connection as DSL (digital subscriber line) and cable modem. This technology holds the transmission of voice for voice control $[17,19,21]$ as well as e-mails and IM (instant messages). Indeed, the received message is analyzed, treated, and the mnemonic keywords are evolved in order to execute the commands.

The infrared light, which is invisible to the human eye, is mainly used in home remote controls to issue unidirectional commands from a distance. The infrared pulses generated by the remote control handset should be expedited to the device through a short and direct line-of-sight. This approach is limited for the case when the transmitter and the receiver are located in short range distance or disposed in the same site.

In contrast, the Bluetooth technology $[8-9,11]$ achieves the data exchange over short distances but the broadcasting of this wireless signal does not require that the controller and the device are facing each other. The range of Bluetooth depends on class of radio used in the implementation. In fact, the most commonly commercially used radio is of class 2 of power $2.5 \mathrm{mw}$ that enables a range of $10 \mathrm{~m} \mathrm{[4].} \mathrm{The} \mathrm{Bluetooth} \mathrm{main} \mathrm{advantages}$ over the other wireless modalities are that PC, mobile phone, android, and i-devices are supporting it. Moreover, the implementation of the Bluetooth module at the control main board provides a soft access and allows the control of appliances.

The SMS (Short Message Service) [7, 16] control requires generally mobile communication systems. The proposed appliances are controlled by a main control board that has the capability to identify the specified number from which the SMS is sent, and therefore classify the mobile numbers that have the priority to access to the automated system. The GSM (Global System for Mobile communication) model is a hardware circuit involved by a SIM (Subscriber Identify Model) card that receives the text massages. Usually, the message is decoded, transmitted to the processing unit based on microcontroller [23-24] that, in turn, activates the relays in order to toggle the current states of the appliances' switches.

The Web method requires a Web server PC connected to microcontroller [7, 13] or PLC (Programmable Logic Controller) [20]. The operations are done from a far distance through TCP/IP (Transmission Control Protocol/Internet Protocol) connection. The system can be mastered from handheld devices or mobile phones with android applications. The great advantages of similar approach are the susceptibility to recognize and to have knowledge about the circumstance of the appliances but the internet connection should be constantly maintained. An alternative manner to realize home 
control via internet is by sending electronic mail [7]. The appropriate configuration of Microsoft Outlook ascertains a specific processing of the e-mail text words and acts according to the present keywords in the e-mail. Unfortunately, the Web intelligent system could be a target for threats and viruses which expose it to security breaches.

The home system based on Wi-Fi technology $[10,15]$ entails a web server PC provided by Wi-Fi card. The hardware control is interfaced with Wi-Fi module that carries out full communication between the PC server and the hardware components up to distance range of $20 \mathrm{~m}$. The commands are sent to the PC server through the internet from other PC or application software installed on smart phones. In spite of the advantages of the Wi-Fi technologies concerning the range and the security, the internet connection should always be retained.

\subsection{Security and Home System Improvements}

The mentioned approaches represent the major categories of techniques adopted in practice as well as those proposed in recent researches. However, many variants were continuously suggested with diversified alternatives to solve many technical problems or to improve failure or insufficiency of others. The control by voice, the security authentication for accessing approved by biometric physiology [6] such as fingerprint, hand geometry, iris, and voice recognition; the protection against hackers for web server control way by equipping sophisticated firewalls and installing dedicated antivirus programs are all examples of upgrading. For domestic security control, smoke, fire and gas detectors realize the home safety as well as intruder detection. Indeed, home systems can be easily connected to the police station, firefighter, hospitals, health care stations, and emergency agencies. Moreover, the wiring assemblage can be planned in a way to isolate appliances against power failure prone or disconnect specific actuator when dysfunction work is remarked.

\subsection{Devices Installation and Control Way Mode}

The cost of the automation system depends on many factors: home size, equipment, components, furniture, custom installations, mode of control, and whether the building is with existing wiring system or is recently constructed. The system can use one master board to control the entire system which is beneficial in case of structure of existing wire. Some systems offered multiple master boards that classify the appliances according to location (bedrooms, garage, kitchen ...) or types (HVAC, security system, domestic appliances ...). This hybrid system is suitable for both kinds of buildings. The third technique is compatible for new building design where the wirings are reduced to a minimum. A network or mesh of distributed points is conceived where each point acts as a host that receives orders and transmits it properly. Moreover, it should take into consideration whether the implemented smart system works only automatically or functions with both manual and automatic modes. Besides, the user should be informed if his system is prone to interference issues with neighboring home systems.

\section{Home Automation using XBee for Elders}

In this study, we are designing a smart home that is intended in particular to elderly, handicapped, decrepit, cripple and disable people. It enables these kinds of residents to control and operate an assortment of appliances. Our attempt offers suitable system that develops easy solutions to be applied to impaired individuals.

The proposed system is specialized for pre-existing houses despite of its eligibility to be extended to new houses. The main idea is that the target persons, particularly the elderly, are living at home most of the time. Since the end users are constantly residing in their dwelling, the web server mode and the GSM mode aren't efficient while the 
conventional Bluetooth system that operates at $10 \mathrm{~m}$ may not cover all the domicile area. The concept of our suggested project is to use wireless communication using XBee transceivers [17] that achieves the total control between the remote control tool and the master main board. The latter is based on microcontroller commanding relays that toggle the current states of the appliances switches.

\subsection{Remote Control Unit}

Various technologies are offered by the e-home community and presented by the manufacturers starting from peculiar software installed on PC or Laptops to dedicated applications on phone mobiles, android devices, and i-devices; from touch screen, keypad to apparatus supported by buttons and switches. In fact, the disabled people could find idevices with touch screen control panel confusing and difficult to use. Therefore, they prefer a simpler remote control using laser-engraved backlit buttons, some switches, and equipped with a LCD screen to display necessary notifications. To each command button is associated a warning LED light that visualizes the situation status of the corresponding appliance. All operations are governed by a microcontroller where the EEPROM memory gives the opportunity to lock the remote by means of pass code stored in its memory.

\subsection{Master Control Board}

As previously mentioned, the proposed home is assumed to have pre-existing wiring which allows the user to use one master control board. Nevertheless, multiple master boards could be configured in order to group the variety of appliances according to location or types. However, the master control board is based on microcontroller interfaced with XBee transceiver module to ensure full communication between the remote control and its base. The microcontroller pins are connected to relays and sensors where the configurations of the On/Off functionality of the demanded appliances are performed according to conditions specified by input sensors.

\subsection{XBee transceivers}

The XBee transceiver module, Series 2, allows creating complex mesh networks based on Zig-Bee firmware. It allows a very reliable and simple communication between microcontrollers through serial port data transfer. The XBee features $(2 \mathrm{~mW}$ output, 120 $\mathrm{m}$ range, built-in antenna, $250 \mathrm{kbps}$ max data rate, and 8 digital IO pins) are convenient for our purpose. Moreover, XBee is supported by point-to-point communication adequate for using one master board and corroborative also by multi-point network compatible for using multiple master boards. The configuration of XBee is simpler where that connected to the remote control runs as server whereas that linked to master board fills in host mode. The XBee characteristics give immunity against interference from neighboring systems and avoid the interaction of closer systems which averts the interruption in their services.

\subsection{Electronic circuits}

The realized prototype is designed to control 8 different appliances: home lights, garden lights, HVAC system, entrance lock door, security system, emergency system, heater, and houseplant watering. However, the system can be extended for larger number of operations. The relays (Figure 1) are considerably adopted to let the low power control signal generated by the microcontroller operate high power devices. The diode connected in reverse bias mode aims to protect the transistor against inductive loads that can damage it due to their back electro-motive force. Moreover, relays can be replaced by Triac that can be suitably applied to control AC power elements such as motor speed control, light dimmers, and temperature control. 


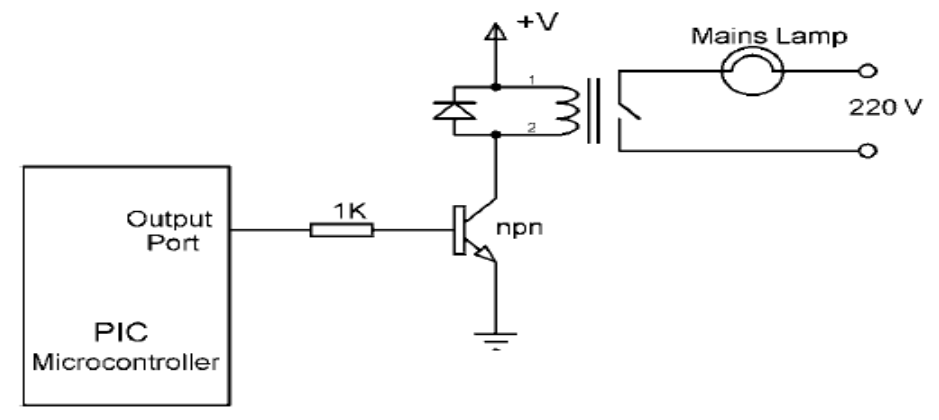

Figure 1. Commanding the On/Off Orders of an Appliance Using Relay

\section{Home Automation System Implementation}

The designed system is conceived by means of a remote control device that sends orders wirelessly using XBee transceivers and by the master board that receives these command signals and activates appliances by triggering the associated electronic relays to achieve the ON/OFF functionality. As we mentioned, the remote control consists of an LCD display for notification messages, many intimation LEDs and eight command buttons for the different appliances, where each button corresponds to a specific appliance. Based on a social point of view, buttons are suitable for the categories of people that are intended to employ this system. Nevertheless, buttons could be replaced by touch screen device for individuals that have an increase knowledge and familiarity with similar visual display screens. Whereas the master board is introduced into a wall position in such a way it ascertains the capability to reach the target appliances. Indeed, the remote control and the master board are intercommunicating to exchange information concerning which appliances are selected to be commutated ON or OFF, as well as the status of the selected appliances sent backward from master board to the remote control in the form of text messages. The XBee transceivers are picked out to ensure secure data exchange without interference and to be immune against intruders and hackers. In fact, the XBee configuration phase required the knowledge of some specific id code that realizes certain security levels.

The procedure of functionality of the home automation system specialized for elders and people with disabilities can be summarized by the following steps. When a button particular to a specified appliance is pressed, an alert LED assigned to this appliance is illumined and an alert message is shown on LCD. Moreover, the remote control sends RF command signals to the master board to be inquired about the new orders. Then, the master board processes the received signal, and the complete treatment will acquaint the microcontroller about the corresponding appliance and the associated pin ports from which the trigger signal should be initiated. When the switching circuit is activated by the microcontroller signal, it turns ON or OFF the appliance according to its previous status. Finally, the master board will broadcast, in a periodic elapsing time, the situation and the position of the appliances being working to be displayed on the LCD of the remote control.

The entire system was completely built using wooden home prototype where the home appliances are modeled by small fan and a group of lights. The software was tested to ensure that the program code doesn't include bug errors and doesn't produce unexpected results. However the system hardware is realized and implemented and the demo validates our system design and shows that the complete operations are executed correctly.

\section{Conclusion and Future Perspectives}

Home automation systems had progressively developed as an important field of control systems. The implementation of such systems continuously increased especially with the 
tendency to standardize their processes. In fact, the capability of devices of different kinds and issuing from various manufacturers to cooperate, communicate and function with high levels of harmony becomes an important factor. Moreover, advanced processes and numerous techniques that are presented intend to reduce the prices of smart home, make the integrated system easier and handled with simplicity, and to achieve permanent degrees of security.

The proposed home automation system is dedicated for elderly, people with disabilities, handicapped persons and others. It consists of remote control supported by command buttons and provided by alert LEDs and a LCD for showing messages. The unique master board toggles the ON/OFF switches of the appliances by means of relays. The remote control and its base are communicating with RF signals realized by XBee transceivers.

In spite of designing our system for individuals that may require load efforts to move, it can be scalable for other users with the ability of appending multiple functionalities and various modalities. However, it can be also adopted in hospitals, health care centers and hospices.

Besides the secure communications achieved by the XBee transceivers, another advantage of this technology resides in the characteristics of putting the system in sleep mode when it is unused for a period and then awaking it when commands are induced. This feature is of great interest since it ascertains an energy saving option and low power consumption.

\section{References}

[1] H. Frank and N. Stoops. Census 2000 special reports, series CENSR-4, Demographic trends in the $20^{\text {th }}$ century, (2002), November.

[2] World population ageing 2013, United Nation UN department of economic and social affairs, populatuion division, (2013).

[3] The official website of the Smart Home Association of Netherlands: http://www.smart-homes.nl/.

[4] The official website of the Bluetooth technology: http://www.bluetooth.com/.

[5] R. Man Li. The usage of automation system in Smart Homr to provie a sustainable indoor environment: A content analysis in Web 1.0. Internaltional Journal of Smart Home, (2013) July, Vol. 7, No. 4, pp. 4759.

[6] B. El-Basioni, S. Abd El-kader, and M. Fakhreldin. Smart home design using wireless sensor network and biometric technologies. International journal of application and innovation in Engineering \& Management (IJAIEM), (2013) March, Vol. 2, Issue 3, pp. 413- 429.

[7] K. Balasubramanian and A. Cellatoglu. Selected Home Automation and Home Security Realizations: An Improved Architecture, Smart Home Systems, Mahmoud A. Al-Qutayri (Ed.), ISBN: 978-953-307050-6, (2010) February.

[8] N, Sriskanthan, F. Tan, and A. Karande. Bluetooth based home automation system. Microprocessors and microsystems, (2002) May, Vol. 26, pp. 281-289.

[9] R. Ramlee, M. Leong, R. Singh, M. Ismail, M. Othman, H. Sulaiman, M. Misran, and M.M. Said. Bluetooth remote home automation system using android application. The international journal of Engineering and Science (IJES), (2013), Vol. 2, Issue 01, pp. 149-153.

[10] S. Kumar. Ubiquitous smart home system using android application. International journal of computer networks \& communcations (IJCNC), (2014) January, Vol. 6, No. 1, pp. 33-43.

[11] S. Panth, and M. Jivani. Home automation system (HAS) using android for mobile phone. International journal of electronics and computer science engineering, (2011) January, Vol. 3, No. 1.

[12] D. Javale, M. Mohsin, S. Nandanwar, and M. Shingate. Home automation and security system using Android ADK. International journal of electronics communication and computer technology (IJECCT), (2013) March, Vol. 3, Issue. 2, pp. 382-385.

[13] Z. Ahmed, M, Ali, and S, Majeed. Implementing computerized and digitally mobile home automation system towards electric appliance control and security system. International Journal of Emergency Science, (2011) September, Vol. 1, No. 3, pp. 487-503.

[14] R. Robles, and T. Kim. Applications, systems and methods in smart home technology: a review. International journal of advanced science and technology, (2010) February, Vol. 15, pp. 37-47.

[15] A. ElShafee, and K. Hamed. Design and implementation of a WIFI based home automation system. World academy of science, engineering and technology, (2012) August, Vol. 6, pp. 1852-1858. 
[16] M. Khiyal, A. Khan, E. Shehzadi. SMS based wireless home appliance control system (HACS) for automating appliances and security. Issues in informing science and information technology, (2009), Vol. 6, pp. 887-894.

[17] Y. Krishna, and S. Nagendram. Zigbee based voice control system for smart home. International journal of computer technology \& applications, (2012) February, Vol. 3, No. 1, pp. 163-168.

[18] I. Kaur. Microcontroller based home automation system with security. International journal of advanced computer science and applications, (2012) December, Vol. 1, No. 6, pp. 60-65.

[19] D. Thakur, and A. Sarma. Voice recognition wireless home automation system based on Zigbee. Journal of electronics and communication engineering (IOSR-JECE), (2013) June, Vol. 6, Issuue 1, pp. 65-75.

[20] O. Bingol, and K. Tasdelen. Web-based smart home automation: PLC controlled implementation. Acta polytechnica Hungarica, (2014), Vol. 11, No. 3, pp. 51-63.

[21] K. Dutta, P. Rai, and V. Shekhe.r. Microcontroller based voice activated wireless automation system. International journal of electrical, electronics \& communication engineering, (2012), Vol. 2, No. 8, pp. 642-649.

[22] B. Hamed. Design \& implementation of smart house control using LabView. International journal of soft computing and engineering, (2012) January, Vol. 1, Issue 6, pp. 98-106.

[23] D. Smith, PIC in practice. A project - based aproach, 2nd ed, News Elsevier (2006)

[24] M. Mazidi, R. Mckinlay, and D. Causey, PIC microcontroller and embedded systems, 1st ed, Prentice Hall (2007).
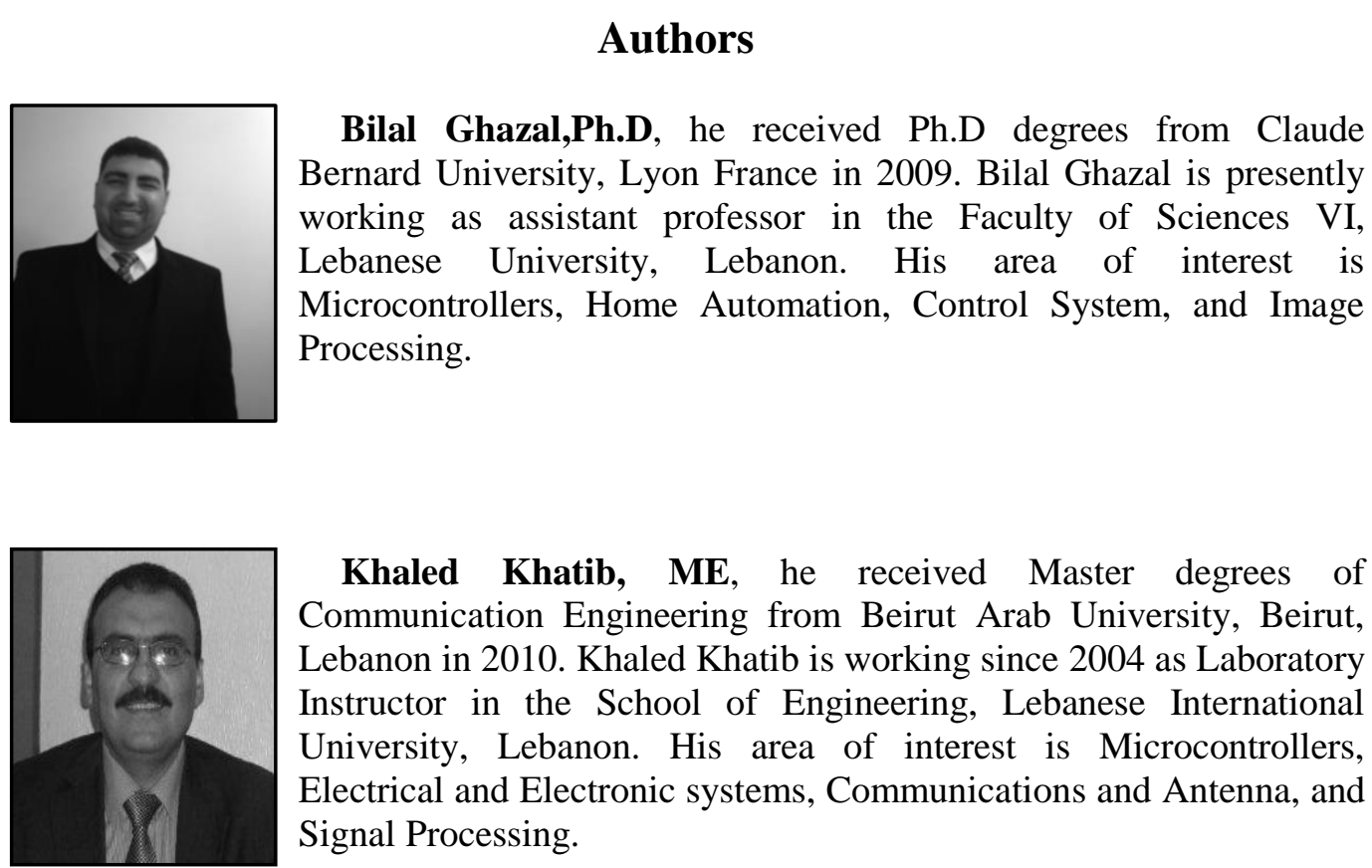

Khaled Khatib, ME, he received Master degrees of Communication Engineering from Beirut Arab University, Beirut, Lebanon in 2010. Khaled Khatib is working since 2004 as Laboratory Instructor in the School of Engineering, Lebanese International University, Lebanon. His area of interest is Microcontrollers, Electrical and Electronic systems, Communications and Antenna, and Signal Processing. 